\title{
Inhibition of miR-19a protects neurons against ischemic stroke through modulating glucose metabolism and neuronal apoptosis
}

\author{
Xiao-Li Ge1 , Jin-Li Wang ${ }^{1}$, Xin Liư ${ }^{2}$ Jia Zhang ${ }^{3}$, Chang Liư and Li Guo ${ }^{4 *}$
}

$\overline{\text { *Correspondence: guoli_med@126. }}$ com

'Department of Neurology, The Second Hospital of Hebei Medical University, Shijiazhuang 050000, China

Full list of author information is available at the end of the article

\section{Abstract}

Background: Accumulating evidence has shown that altered microRNA (miR) modulation is implicated in the pathologies of ischemic stroke. However, it is unclear whether and how hsa-miR-19a-3p mediates cerebral ischemic injury. Herein, we investigated the functional role of miR-19a-3p in cerebral ischemic injury and explored its underlying regulatory mechanism.

Methods: In vivo ischemic/reperfusion (I/R) neuronal injury and in vitro oxygenglucose deprivation (OGD) were established. Expression of miR-19a-3p was determined by quantitative real-time polymerase chain reaction (qRT-PCR). Glucose uptake, lactate production, and apoptosis were determined. ADIPOR2 was predicted as a target of miR$19 a-3 p$ in silico and experimentally validated by qRT-PCR, Western blot analysis and luciferase assay assays.

Results: MiR-19a expression was significantly downregulated and upregulated in rat neurons and astrocytes, respectively $(P<0.01)$. A significantly elevated level of miR-19a$3 p$ was found in $I / R$ and OGD models in comparison to sham/control groups $(P<0.01)$. Expression of the glycolysis enzyme markers LDHA, PKM2, HK2, Glut1 and PDK1, apoptosis-related factors levels, apoptosis, glucose uptake, and lactate production were significantly repressed by both $I / R$ and $O G D(P<0.01$ in each case). Moreover, miR-19a$3 p$ mimic aggravated, while miR-19a-3p inhibitor alleviated, the above observations. Adipor2 was predicted and confirmed to be a direct target of miR-19a. Furthermore, restoration of Adipor2 reversed miR-19a-3p-induced effects.

Conclusions: Collectively, our results indicate that elevated miR-19a-3p mediates cerebral ischemic injury by targeting ADIPOR2. MiR-19a-3p attenuation thus might offer hope of a novel therapeutic target for ischemic stroke injury treatment.

Keywords: Ischemic stroke, miR-19a-3p, ADIPOR2, Glucose metabolism, Apoptosis

\section{Introduction}

Brain ischemic stroke is known as the commonest cerebrovascular disease and a primary public health problem, characterized by a high incidence and mortality, acute onset, rapid development and severe outcomes, and unfortunately, its incidence is on the rise [1-3]. Cerebral ischemic injury, identified as the fundamental pathophysiological basis of ischemic stroke, has a complicated pathogenesis concerning multiple biological

(C) The Author(s). 2019 Open Access This article is distributed under the terms of the Creative Commons Attribution 4.0 International License (http://creativecommons.org/licenses/by/4.0/), which permits unrestricted use, distribution, and reproduction in any medium, provided you give appropriate credit to the original author(s) and the source, provide a link to the Creative Commons license, and indicate if changes were made. The Creative Commons Public Domain Dedication waiver (http://creativecommons.org/ publicdomain/zero/1.0/) applies to the data made available in this article, unless otherwise stated. 
events, such as neuronal apoptosis $[4,5]$, astrocyte activation [6, 7], proinflammatory reaction and oxidative stress $[8,9]$. Cerebral ischemia results in brain impairment on account of deprivation of oxygen and glucose resulting from blockage of local blood supply. In spite of substantial advances in cerebral ischemic injury therapeutic strategies, the therapeutic effect is still far from ideal for a large number of patients, which is possibly due to a lack of clear understanding of the pathological process [10-12]. Thereby, delineating the molecular mechanism of ischemic stroke is urgent to develop effective therapies for cerebral ischemic injury.

MicroRNAs (miRs), 21-22 nucleotides long, are short noncoding RNAs and are known to function in post-transcriptional regulation of gene expression through majorly binding the 3 '-untranslated region (UTR) of their target messenger RNA (mRNA), accordingly modulating cells' behaviors [13, 14]. The broadly conserved miR-19a-3p, a crucial component of the miR-17-92 cluster, has been shown to be involved in pathogenesis/disease progression in lung cancer [15], gastric cancer [16], breast cancer [17], and hepatocellular carcinoma [18]. Furthermore, miR-19a-3p has been found to be overexpressed in glioma cells and astrocytic glioma tissues and its overexpression is strongly linked with malignancy grades in glioma patients, indicating an important role in gliomagenesis [19]. MiR-19a-3p is increased and positively correlated with poor survival in astrocytoma patients [20]. MiR-19a-3p has been shown to stimulate axonal outgrowth in embryonic cortical neurons [21]. Importantly, the miR-17-92 cluster is upregulated in neural progenitor cells of mouse, and its overexpression observed either in cultured ischemic neural progenitor cells or in the subventricular zone of ischemic animals markedly accelerated cell proliferation, while repression of individual components of the miR-17-92 cluster, miR-18a and miR-19a-3p, inhibited cell proliferation and enhanced cell death [22]. However, these studies have largely been correlative and the role of miR-19a-3p in cerebral ischemic injury has not been investigated; hence it was investigated in I/R and OGD models in the current study.

\section{Materials and methods}

\section{Isolation and culture of neuronal cells and astrocytes}

Primary neurons were isolated from newborn Sprague Dawley (SD) rats. First, the hippocampal tissues were digested by $0.125 \%$ trypsin for $10 \mathrm{~min}$. After separation and centrifugation, the obtained neurons were inoculated into 6-well plates after the wells were treated by poly-d-lysine (Sigma-Aldrich, St. Louis, MO, USA) for $12 \mathrm{~h}$. The cells were maintained using neurobasal medium containing with $2 \% \mathrm{~B} 27,1 \%$ glutamine and cytarabine at $37^{\circ} \mathrm{C}$ with $5 \% \mathrm{CO}_{2}$. To establish the oxygen-glucose deprivation (OGD) model, neurons were cultured in deoxygenated, glucose-free Hanks' Balanced Salt Solution at $37^{\circ} \mathrm{C}$ with $5 \% \mathrm{CO}_{2}$ and $95 \% \mathrm{~N}_{2}$ for $24 \mathrm{~h}$. Then the cells were cultured under normoxic conditions.

Primary astrocytes were isolated from newborn SD rats. The neocortices were treated using $0.09 \%$ trypsin for $25 \mathrm{~min}$ after they were dissected. The single cells obtained after centrifugation were inoculated into 6-well plates treated by poly-d-lysine (Sigma-Aldrich). Eagle's Minimal Essential Medium was used to culture cortical astrocytes with 10\% FBS, $21 \mathrm{mM}$ glucose and 10\% EGF (Gibco, Grand Island, NY, USA). Cells were cultured at $37^{\circ} \mathrm{C}$ with $5 \% \mathrm{CO}_{2}$. 


\section{Rat ischemic stroke model establishment}

The Ethics Committee of the Second Hospital of Hebei Medical University has reviewed and approved this study. All the experimental procedures were carried out according to the United States National Institutes of Health guidelines for the use of experimental animals. Rat ischemic stroke models were established by performing middle cerebral artery occlusion in adult (8-10 weeks) male Sprague Dawley (SD) rats weighing $200 \pm 12 \mathrm{~g}$. All rats were anesthetized using sodium pentobarbital with a concentration of $100 \mathrm{mg} / \mathrm{kg}$. Cervical incision was performed to expose arteries. The right internal carotid artery and middle cerebral artery were ligated by a nylon suture for 90 $\mathrm{min}$. Then all rats were recovered for $24 \mathrm{~h}$ before being sacrificed. 2,3,5-triphenyltetrazolium chloride (TTC) staining was performed using routine protocols to confirm successful ischemia induction (Fig. 1a). Ischemia induction was confirmed by a blinded pathologist by reviewing the TTC staining of the experimental groups.

\section{Quantitative real-time polymerase chain reaction (qRT-PCR)}

Total RNA was extracted using Trizol (Invitrogen, Carlsbad, California, USA). To generate cDNA, the Mir-X miRNA FirstStrand Synthesis Kit and the Reverse Transcriptase Kit (Takara, Dalian, China) were used. The qPCR was carried out using a SYBR Premix Ex Taq TM II (Takara) with the ABI 7900 qRT-PCR System (Applied Biosystems, Foster City, CA, USA). The primer sequences used were as follows: mature rno-miR-19a-3p stem-loop PCR (product size: $71 \mathrm{bp}$ ): F: 5'-TGTGCAAATCTATGCAAA-3', R: 5'-CAGTGCGTGT CGTGGAGT-3'; U6: F: 5'-CGCAAGGATGACACGCAAAT-3', R: 5'-ATTTGCGTGTCA TCCTTGCG-3'; Adipor2 (product size: $140 \mathrm{bp):} \mathrm{F:} \mathrm{5'-GGAGTGTTCGTGGGCTTG}$ GG-3', R: 5' - GCAGCTCCTGTGATATAGAGG-3'; Gapdh (product size: 177 bp): F: 5' ATGACATCAAGAAGGTGGTG-3'; R: 5'-CATACCAGGAAATGAGCTTG-3'. U6 and Gapdh were used for normalization of miR-19a-3p and Adipor2 expression. Data were analyzed with the $2^{-\Delta \Delta \mathrm{Ct}}$ method and expressed as folds over experimental control groups.

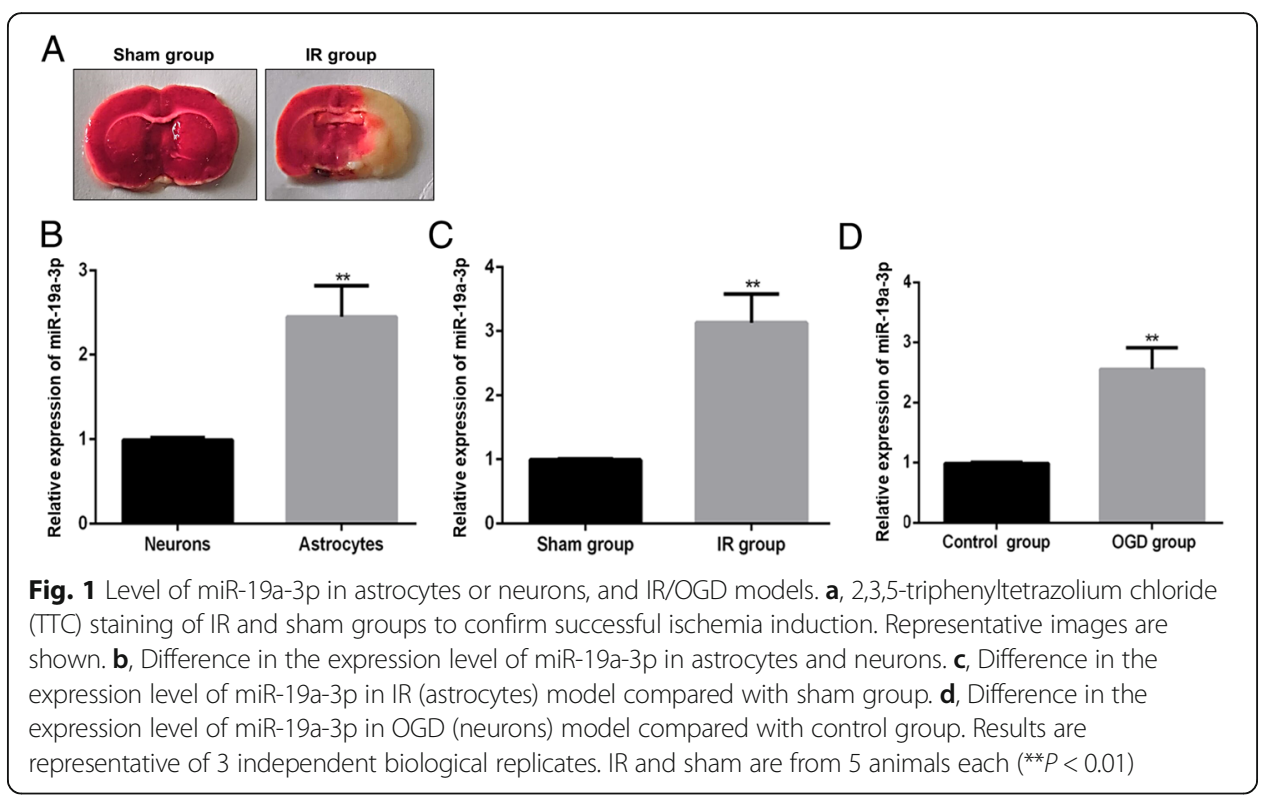




\section{Western blot}

Total proteins were isolated using RIPA lysis Buffer (Beyotime, Shanghai, China). Protein concentrations were tested using a bicinchoninic acid (BCA) kit (Beyotime). Twenty $\mu \mathrm{g}$ of proteins were resolved by sodium dodecyl sulphate-polyacrylamide gel electrophoresis (SDS-PAGE). Membranes were probed with primary antibodies against LDHA (1: 1000, Abcam, Cambridge, USA), PKM2 (1: 1000, Abcam), HK2 (1: 1000, Abcam), Glut1 (1: 1000, Abcam), PDK1 (1: 1000, Abcam), Bim (1: 1000, Abcam), Bax (1: 1000, Abcam), caspase-9 (1: 500, Abcam), ADIPOR2 (1: 1000, Abcam), and GAPDH (1: 1000, Beyotime). GAPDH was used as the loading control protein. Densitometry analyses was performed using Image J software.

\section{Transfection}

Cells $\left(5 \times 10^{5}\right.$ cells/well $)$ were transfected with miR-19a-3p mimic or inhibitor of miR-19a-3p (Shanghai GenePharma Co., Ltd., Shanghai, China), and their controls using Lipofectamine 2000 (ThermoFisher Scientific, Shanghai, China). Cells were harvested after $48 \mathrm{~h}$ of transfection to perform follow-up experiments as indicated.

\section{Luciferase assay}

The potential targets of miR-19a-3p were in silico predicted using TargetScan software. The wild or mutant (miR-19a-3p seed deleted (596-603)) of the 3'-UTR of Adipor2 was cloned into the pmiRGLO vector (Promega, Madison, WI, USA). The miR-19a-3p mimic and its control were transfected into neuronal cells. After $48 \mathrm{~h}$ of transfection, the relative luciferase activities of cells were measured by the Dual-Glo Luciferase Assay System (Promega) in accordance with the manufacturer's introductions. Renilla luciferase activity was used to normalize luciferase activity.

Terminal-deoxynucleotidyl transferase-mediated UTP nick end labeling (TUNEL) staining Cell apoptosis was tested by TUNEL staining using the One Step TUNEL Apoptosis Assay Kit (Beyotime) following the manufacturer's guidelines. After TUNEL staining, cells were incubated with DAPI. Cells were imaged using a fluorescence microscope (Fluoview FV1000, Olympus, Tokyo, Japan) and counted under five random fields. Neuronal apoptosis was assessed by the percentage of TUNEL-positive cells from the total number of cells (DAPI- positive cells).

\section{Glucose metabolism assays}

For determining the change of glucose metabolism, the uptake of glucose and production of lactate analyses were assessed by the Glucose Uptake Colorimetric Assay Kit and Lactate Colorimetric Assay Kit (Sigma-Aldrich, St Louis, MO, USA) following the manufacturer's protocol.

\section{Statistical analysis}

Statistical analysis was conducted using SPSS Statistics software 22.0 (Chicago, IL, USA). Data are represented (except otherwise mentioned) as mean \pm standard deviation (SD). The differences between two groups were analyzed using Student's $t$-test. The 
differences of multiple groups were performed by one-way ANOVA followed by post-hoc Dunnett $t$ test. $P<0.05$ was considered as statistically significant.

\section{Results}

Low expression of miR-19a-3p in rat neurons and miR-19a-3p upregulation in $\mathrm{I} / \mathrm{R}$ and OGD models

We initially determined whether basal miR-19a-3p expression was different in neuronal cells and astrocytes, two important components affected in ischemic brain injury. MiR-19a-3p level was significantly lower in neurons compared to astrocytes (Fig. 1b; *: $P<0.01)$. However, induction of $\mathrm{I} / \mathrm{R}$ in vivo in astrocytes or OGD in vitro in neuronal cells significantly induced miR-19a-3p expression compared to the sham or control group, respectively, and it was up-regulated in I/R and OGD models compared with sham/control groups (Fig. $1 \mathrm{c}$ and $\mathrm{d},{ }^{* *} P<0.01$ ), highlighting that miR-19a-3p might either be involved in or serve as an effector of ischemic brain injury.

\section{Glucose metabolism is repressed by IR/OGD treatment}

It is well known that ischemic brain injury results in deprivation of cellular energy reserve leading to death in cells. We thus assessed expression levels of glycolysis enzyme-associated factors LDHA, PKM2, HK2, Glut1 and PDK1, glucose uptake and lactate production after IR/OGD. LDHA, PKM2, HK2, Glut1 and PDK1 expression (Fig. 2a, d), glucose uptake (Fig. 2b, e) and lactate production (Fig. 2c, f) were significantly decreased in astrocytes within the IR and neuronal cells within the OGD group $(* * P<0.01$ in each case). These results indicate that glucose metabolism rescue aids in ameliorating ischemic brain injury.

\section{Neuronal apoptosis is induced by OGD treatment}

For detecting neuronal apoptosis induced by OGD, apoptosis and levels of apoptosis-related proteins were measured using TUNEL and western blot analyses, respectively. TUNEL-stained cells were significantly more numerous in the OGD group compared to the control group (Fig. $3 \mathrm{a}$; ${ }^{* *} P<0.01$ ). Concomitantly, the apoptosis markers Bax, Bim and caspase- 9 were detectably upregulated in OGD neurons, but not in controls (Fig. 3b; ${ }^{*} P<0.01$ ). Expression of pro- and cleaved-caspase-3 confirmed increased apoptosis in the OGD treatment groups (Fig. 3c).

\section{MiR-19a inhibits glucose uptake and promotes neuronal apoptosis by targeting Adipor2}

To determine the linkage between miR-19a-3p expression and glucose metabolism or neuronal apoptosis in OGD, miR-19a-3p mimic or inhibitor was transfected to neuronal cells in the OGD and control groups. Successful miR-19a-3p overexpression and knockdown by the mimic and inhibitor, respectively, was confirmed (Fig. $4 \mathrm{a},{ }^{* *} P<0.01$ ). Expression of glycolysis enzymes, glucose uptake and lactate production were markedly reduced after miR-19a-3p mimic transfection and were rescued when upregulated in OGD neurons (Fig. $4 \mathrm{~b}-\mathrm{d}$; ${ }^{* *} P<0.01$ ). Inhibition of miR-19a-3p by the inhibitor downregulated both apoptosis (Fig. 4e) and expression of pro-apoptosis markers (Fig. 4f-g),

To investigate downstream targets of miR-19a-3p that might be responsible for its role in ischemic injury, we used TargetScan software to predict putative targets. There 


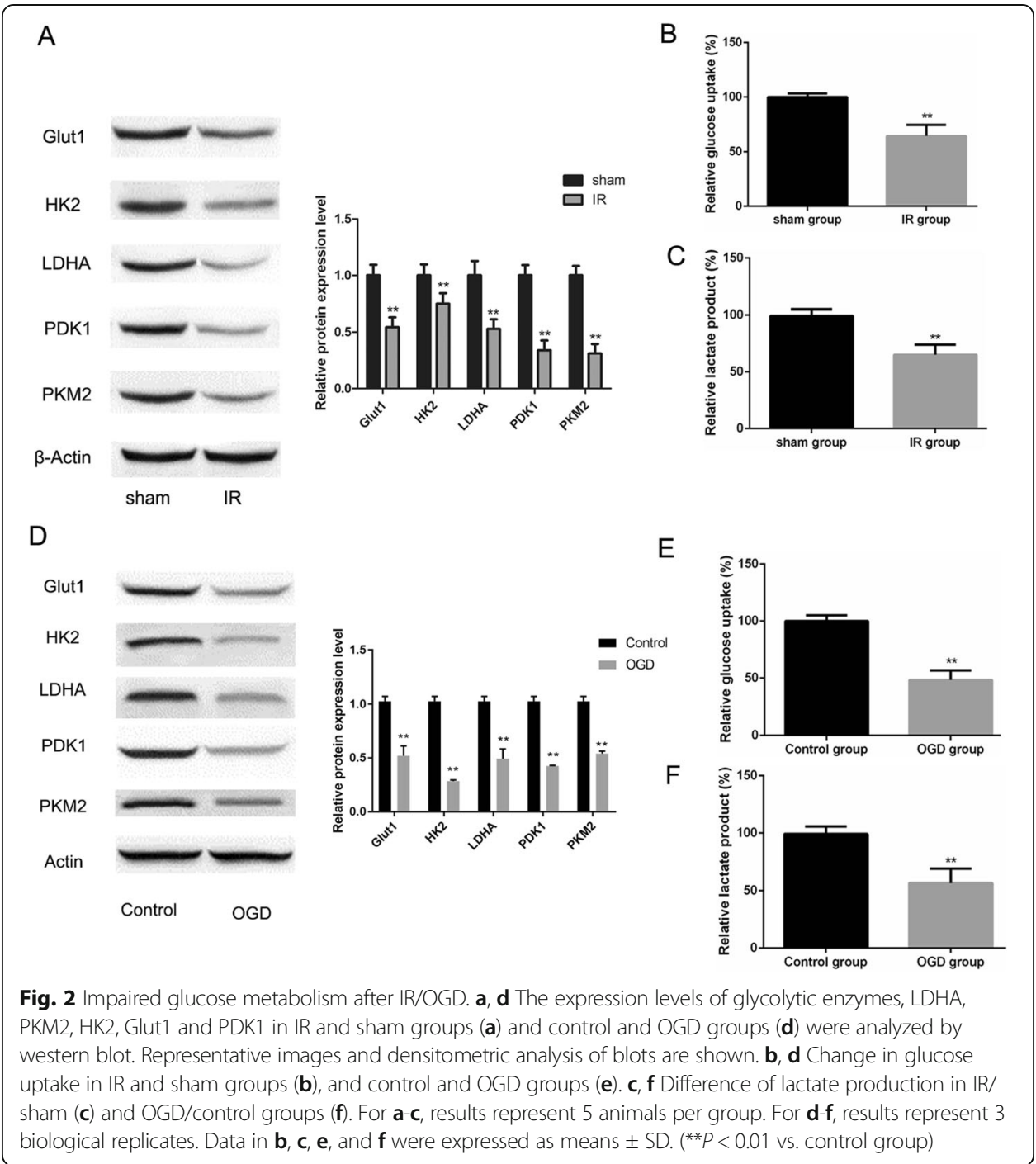

were more than 500 putative miR-19a-3p targets predicted. One of the predicted targets, Adipor-2 (encoding for adiponectin receptor 2), was of particular interest for two reasons. First, adiponectin receptors have been shown to be expressed in specific neurogenic niches and associated with brain repair [23]. Secondly, Adipor-1 has been shown to mediate electroacupuncture mediated cerebral attenuation of cerebral I/R injury in diabetic mice [24]. Hence, we proceeded to determine whether Adipor2 is a direct target of miR-19a-3p and one of the genes post-transcriptionally regulated by miR-19a-3p during cerebral $I / R$ injury. TargetScan predicted that miR-19a-3p targets position 596-603 of human ADIPOR2 and position 653-660 of the rat Adipor2 3'-UTR, which were entirely conserved in the sequence (Fig. 5a, b). Luciferase expression of wild-type Adipor 2 3'-UTR harboring Firefly luciferase ORF was significantly downregulated following transfection of miR-19a-3p mimic (Fig. 5 b; ${ }^{* *} P<0.01$ ). However, luciferase expression of the reporter was not changed following miR-19a-3p transfection in neuronal cells when the miR-19a-3p seed was mutated in the reporter (Fig. 5b), confirming Adipor 2 as a bona fide target of miR-19a-3p. We next investigated whether increased miR-19a-3p in OGD neurons actually results in downregulation of 

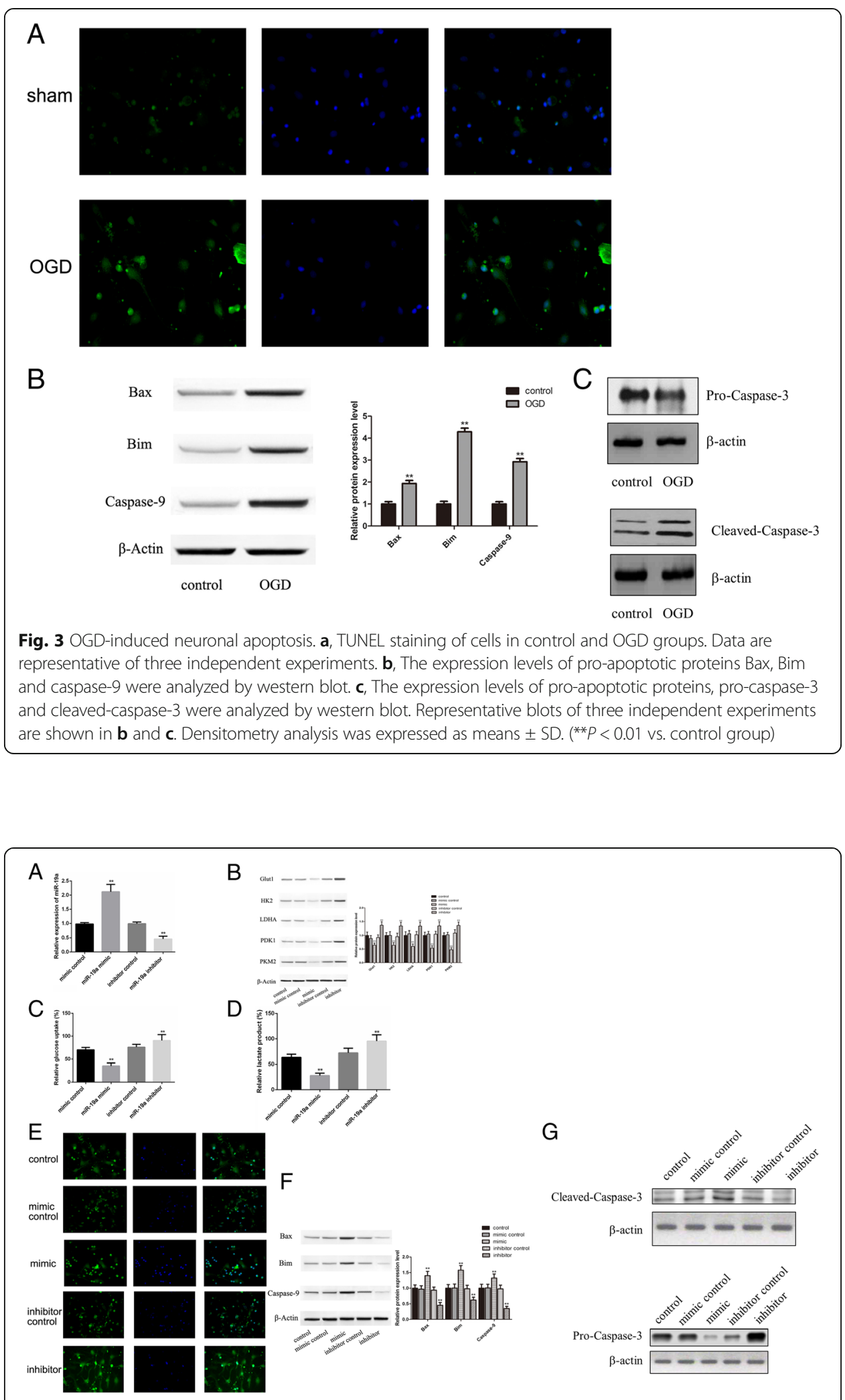

Fig. 4 Effect of miR-19a mimic/inhibitor on glucose metabolism and apoptosis in OGD-treated neurons. $\mathbf{a}$, Successful transfection of miR-19a-3p mimic/inhibitor was verified by qRT-PCR. B-F, The effects of miR-19a-3p on expression of glycolytic enzymes (b), glucose uptake, (c) lactate production (d), and apoptosis (e-g) were assayed as described in Figs. 2 and 3. All data were expressed as means \pm SD. (**P $<0.01$ vs. control group) 


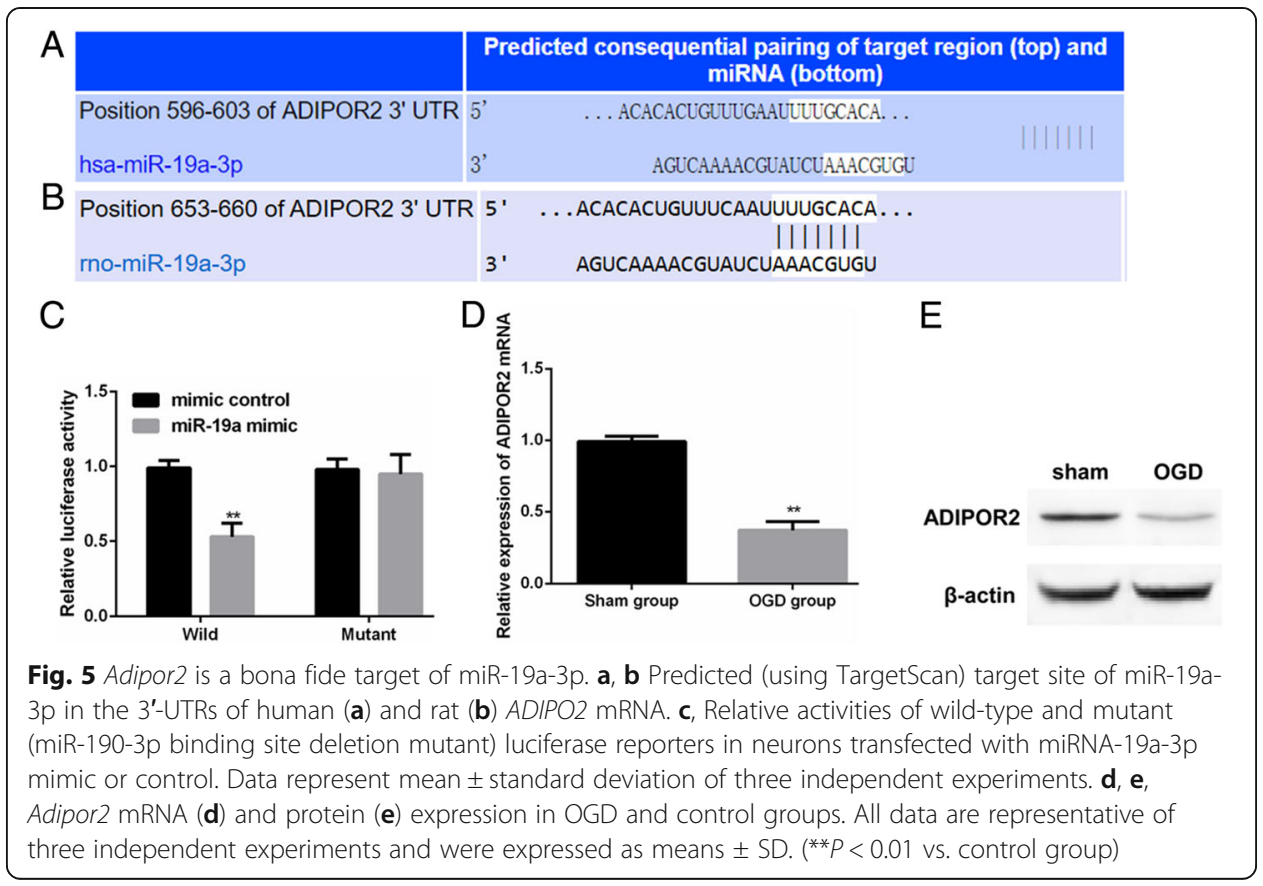

Adipor2. Both Adipor2 mRNA (Fig. 5c) and protein expression (Fig. 5d) was significantly downregulated in OGD neurons compared to the control group. Cumulatively, these results indicated that increased miR-19a-3p in the OGD neuronal cells is targeting and downregulating expression of Adipor2.

\section{ADIPOR2 is the functional effector of miR-19a-3p during cerebral ischemic injury}

Even though our results confirmed that Adipor2 is a bona fide target of miR-19a-3p, it still did not imply that the pathogenic effect of miR-19a-3p during $I / R$ injury is being mediated by ADIPOR2. To ascertain this, we transfected OGD neurons with siRNA targeting Adipor2, either alone or along with the miR-19a-3p inhibitor. Adipor2 silencing in control neurons downregulated glucose uptake and lactate production, while co-transfection with the miR-19a inhibitor mitigated these phenomena (Fig. 6a, b), confirming that the ischemic brain damage mediated by miR-19a-3p is mediated at least in part by downregulation of Adipor2.

\section{Discussion}

In the present study, we observed that miR-19a-3p was higher in I/R and OGD models than controls. Significantly, miR-19a-3p inhibition effectively mitigated IR/OGD-induced repression of glycolysis enzymes' expression, glucose uptake and lactate production, and neuronal apoptosis, which was regulated by targeting Adipor2 (Fig. 6c). Collectively, our observations elucidate one target gene which is downregulated by elevated miR-19a-3p during cerebral ischemic injury pathogenesis.

Astrocytes, identified as the largest number of cells in the central nervous system (CNS), interact with neurons in order to maintain the stability of the CNS environment [25-27]. In response to cerebral ischemia, astrocytes undergo hyperplasia and metabolic reserve enhancement and delayed apoptosis is observed in neurons [28-30]. 


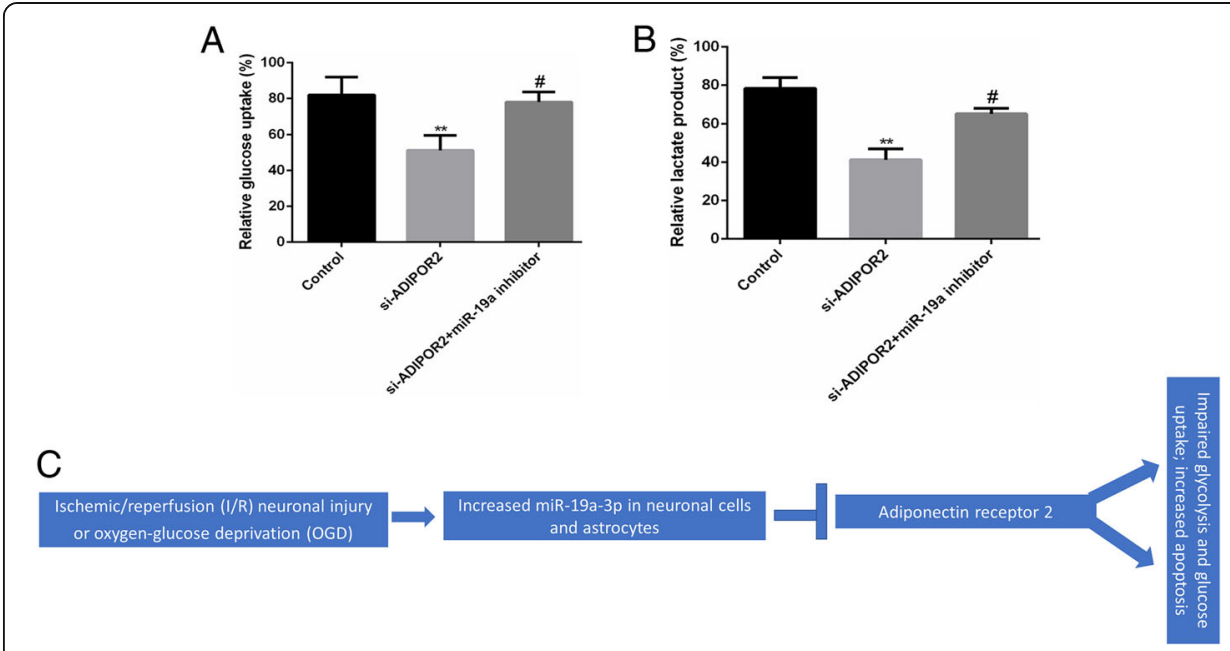

Fig. 6 Adipor2 is an effector of miR-19a-3p's role in inducing cerebral ischemic injury. a, b, Glucose uptake (a) and lactate production (b) in neuronal cells transfected with siRNA-targeting Adipor2 either alone or in combination with anti-miR-19a-3p inhibitor. All data are representative of three independent experiments and were expressed as means \pm SD. ( ${ }^{*} P<0.01$ vs. control group, $\# P<0.05$ vs. si- ADIPOR2 group). c, Model summarizing the major findings of the current study highlighting how elevated miR-19a-3p expression during IR and OGD can result in impaired glucose metabolism and apoptosis by targeting Adipor2

Aberrant miRNA expression levels have been correlated with models of cerebral ischemia injury [31-33]. MiR-19a-3p has been found to highly correlate with the development of a variety of cancers, especially in astrocytoma [20]. Overexpression of miR-19a-3p is observed in astrocytoma patients; in addition, its overexpression occurred in cultured ischemic neural progenitor cells and promoted cell proliferation [22], implying that miR-19a-3p plays a role in regulating neural cell function. One study emphasized the cell-type-specific expression of miR-19a-3p by detecting high expression in astrocytes and a low level in neurons, suggesting a probable impaired effect of miR-19a-3p in preserving neural function.

During cerebral ischemia, nerve cells undergo irreversible damage due to the complete cessation of glucose and oxygen supply to the ischemic core [34-37]. Thus, ameliorating glucose metabolism and controlling neural cell apoptosis is of great importance to improve cerebral ischemia damage [38, 39]. Modulation of miR-19a-3p expression impacted glycolytic enzymes and neuronal apoptosis, even though our results do not indicate the specific downstream effectors that are directly regulating these processes.

ADIPOR2, encoding adiponectin receptor2, has been implicated in cardiovascular diseases [40, 41], fatty liver disease [42], diabetes [43], diabetic nephropathy [44], and bone metabolic disorders [45]. In each of these pathogenic conditions forced overexpression of ADIPOR2 ameliorates the disease process. A correlation between ADIPOR2 expression and ischemic stroke has also been previously established [46]. Our experiments corroborate these findings and highlight the potential need to test whether adenovirus-mediated overexpression of ADIPOR2 will be a viable therapeutic intervention in in vitro and in vivo models of cerebral ischemic injury. In light of recent reports emerging that offer contradictory findings about the dual role of astrocyte and astrocyte-neuron interactions in metabolic alterations accompanied by cerebral ischemia brain injury, further work will be required to investigate the significance of astrocytes. Additionally, the role of other targets of miR-19a-3p need to be evaluated. 


\section{Abbreviations}

BCA: Bicinchoninic acid; CNS: Central nervous system; ECL: Enhanced chemiluminescence; HRP: Horseradish peroxidase; miRs: MicroRNAs; OGD: Oxygen-glucose deprivation model; SD: Sprague Dawley; SD: standard deviation; SDSPAGE: Sodium dodecyl sulphate-polyacrylamide gel electrophoresis; TUNEL: Transferase-mediated UTP nick end labeling; UTR: 3'-untranslated region

\section{Acknowledgements}

Not applicable.

\section{Funding}

This research did not receive any specific grant from funding agencies in the public, commercial, or not-for-profit sectors.

\section{Availability of data and materials}

All data generated or analyzed during this study are included in this published article.

\section{Authors' contributions}

$X L G$ and $L G$ designed and performed this study, and wrote the article. JLW, XL, JZ And CL performed data collection and analysis. All authors read and approved the final manuscript.

\section{Ethics approval and consent to participate}

The Ethics Committee of the Second Hospital of Hebei Medical University has reviewed and approved this study (IACUC-Hebmu-2017129; 2017/12/9). All the experimental procedures were carried out according to the National Institutes of Health guidelines for the use of experimental animals.

\section{Competing interests}

The authors declare that they have no competing interests.

\section{Publisher's Note}

Springer Nature remains neutral with regard to jurisdictional claims in published maps and institutional affiliations.

\section{Author details}

${ }^{1}$ Department of Neurology, The Second Hospital of Hebei Medical University, Shijiazhuang 050000, China. ${ }^{2}$ Department of Neurosurgery, The Second Hospital of Hebei Medical University, Shijiazhuang 050000, China. ${ }^{3}$ Department of Obstetrics, The Second Hospital of Hebei Medical University, Shijiazhuang 050000, China. ${ }^{4}$ Department of Rehabilitation, The Second Hospital of Hebei Medical University, Shijiazhuang 050000, China.

Received: 4 December 2018 Accepted: 15 May 2019

Published online: 31 May 2019

\section{References}

1. Randolph SA. Ischemic stroke. Workplace Health Saf. 2016;64(9):444.

2. Stack CA, Cole JW. Ischemic stroke in young adults. Curr Opin Cardiol. 2018;33(6):594-604.

3. Cabral NL, Nagel V, Conforto AB, Amaral CH, Venancio VG, Safanelli J, Ibiapina F, Longo AL, Zetola VHF. Five-year survival, disability, and recurrence after first-ever stroke in a middle-income country: a population-based study in Joinvile, Brazil. Int J Stroke. 2018;13:725-33.

4. Ye X, Shen T, Hu J, Zhang L, Zhang Y, Bao L, Cui C, Jin G, Zan K, Zhang Z, Yang X, Shi H, Zu J, Yu M, Song C, Wang Y, Qi $\mathrm{S}$, Cui G. Purinergic $2 X 7$ receptor/NLRP3 pathway triggers neuronal apoptosis after ischemic stroke in the mouse. Exp Neurol. 2017:292:46-55.

5. Radak D, Katsiki N, Resanovic I, Jovanovic A, Sudar-Milovanovic E, Zafirovic S, Mousad SA, Isenovic ER. Apoptosis and acute brain ischemia in ischemic stroke. Curr Vasc Pharmacol. 2017;15(2):115-22.

6. Pekny M, Wilhelmsson U, Tatlisumak T, Pekna M. Astrocyte activation and reactive gliosis-a new target in stroke? Neurosci Lett 2018. doi: https://doi.org/10.1016/j.neulet.2018.07.021. [Epub ahead of print].

7. Stary CM, Giffard RG. Advances in astrocyte-targeted approaches for stroke therapy: an emerging role for mitochondria and microRNAS. Neurochem Res. 2015;40(2):301-7.

8. Rana AK, Singh D. Targeting glycogen synthase kinase-3 for oxidative stress and neuroinflammation: opportunities, challenges and future directions for cerebral stroke management. Neuropharmacology. 2018;139:124-36.

9. Belisario AR, Silva CM, Velloso-Rodrigues C, Viana MB. Genetic, laboratory and clinical risk factors in the development of overt ischemic stroke in children with sickle cell disease. Hematol Transfus Cell Ther. 2018;40(2):166-81.

10. Sarkar S, Chakraborty D, Bhowmik A, Ghosh MK. Cerebral ischemic stroke: cellular fate and therapeutic opportunities. Front Biosci (Landmark Ed). 2019;24:435-50.

11. Rodrigo R, Fernandez-Gajardo R, Gutierrez R, Matamala JM, Carrasco R, Miranda-Merchak A, Feuerhake W. Oxidative stress and pathophysiology of ischemic stroke: novel therapeutic opportunities. CNS Neurol Disord Drug Targets. 2013;12(5):698-714

12. Navis A, Garcia-Santibanez R, Skliut M. Epidemiology and outcomes of ischemic stroke and transient ischemic attack in the adult and geriatric population. J Stroke Cerebrovasc Dis 2018. doi: https://doi.org/10.1016/j.jstrokecerebrovasdis.2018. 09.013. [Epub ahead of print].

13. Mohr AM, Mott JL. Overview of microRNA biology. Semin Liver Dis. 2015;35(1):3-11.

14. Saliminejad K, Khorram Khorshid HR, Soleymani Fard S, Ghaffari SH. An overview of microRNAs: biology, functions, therapeutics, and analysis methods. J Cell Physiol 2018. doi: https://doi.org/10.1002/jcp.27486. [Epub ahead of print]. 
15. Zhang X, Li Y, Qi P, Ma Z. Biology of MiR-17-92 cluster and its Progress in lung Cancer. Int J Med Sci. 2018;15(13):1443-8.

16. Li Y, LV S, Ning H, Li K, Zhou X, Xv H, Wen H. Down-regulation of CASC2 contributes to cisplatin resistance in gastric cancer by sponging miR-19a. Biomed Pharmacother. 2018;108:1775-82.

17. Wu J, Jiang Y, Cao W, Li X, Xie C, Geng S, Zhu M, Liang Z, Zhu J, Zhu W, Wu R, Ma X, Huang C, Yang X, Wang S, Zhong C. miR-19 targeting of PTEN mediates butyl benzyl phthalate-induced proliferation in both ER(+) and ER(-) breast cancer cells. Toxicol Lett. 2018;295:124-33.

18. Jiang XM, Yu XN, Liu TT, Zhu HR, Shi X, Bilegsaikhan E, Guo HY, Song GQ, Weng SQ, Huang XX, Dong L, Janssen HLA, Shen XZ, Zhu JM. microRNA-19a-3p promotes tumor metastasis and chemoresistance through the PTEN/Akt pathway in hepatocellular carcinoma. Biomed Pharmacother. 2018;105:1147-54.

19. Jia Z, Wang K, Zhang A, Wang G, Kang C, Han L, Pu P. miR-19a and miR-19b overexpression in gliomas. Pathol Oncol Res. 2013;19(4):847-53.

20. Zhi F, Shao N, Wang R, Deng D, Xue L, Wang Q, Zhang Y, Shi Y, Xia X, Wang S, Lan Q, Yang Y. Identification of 9 serum microRNAs as potential noninvasive biomarkers of human astrocytoma. Neuro-Oncology. 2015;17(3):383-91.

21. Zhang Y, Ueno Y, Liu XS, Buller B, Wang X, Chopp M, Zhang ZG. The MicroRNA-17-92 cluster enhances axonal outgrowth in embryonic cortical neurons. J Neurosci. 2013;33(16):6885-94.

22. Liu XS, Chopp M, Wang XL, Zhang L, Hozeska-Solgot A, Tang T, Kassis H, Zhang RL, Chen C, Xu J, Zhang ZG. MicroRNA-17-92 cluster mediates the proliferation and survival of neural progenitor cells after stroke. J Biol Chem. 2013;288(18):12478-88.

23. Rastegar S, Parimisetty A, Cassam-Sulliman N, Narra Sai S, Weber S, Rastegar M, Viranaicken W, Couret D, Planesse C, Strähle U, Meilhac O, Lefebvre d'Hellencourt C, Diotel N. Expression of adiponectin receptors in the brain of adult zebrafish and mouse: links with neurogenic niches and brain repair. J Comp Neurol 2019. doi: https://doi.org/10.1002/cne.24669. [Epub ahead of print].

24. Guo F, Jiang T, Song W, Wei H, Wang F, Liu L, Ma L, Yin H, Wang Q, Xiong L. Electroacupuncture attenuates cerebral ischemia-reperfusion injury in diabetic mice through adiponectin receptor 1-mediated phosphorylation of GSK-3 3 . Mol Neurobiol. 2015;51(2):685-95.

25. Anderson MA, Burda JE, Ren Y, Ao Y, O'Shea TM, Kawaguchi R, Coppola G, Khakh BS, Deming TJ, Sofroniew MV. Astrocyte scar formation aids central nervous system axon regeneration. Nature. 2016;532(7598):195-200.

26. Kovacs GG. Cellular reactions of the central nervous system. Handb Clin Neurol. 2017;145:13-23.

27. Nortley R, Attwell D. Control of brain energy supply by astrocytes. Curr Opin Neurobiol. 2017:47:80-5.

28. Wang W, Redecker C, Yu ZY, Xie MJ, Tian DS, Zhang L, Bu BT, Witte OW. Rat focal cerebral ischemia induced astrocyte proliferation and delayed neuronal death are attenuated by cyclin-dependent kinase inhibition. J Clin Neurosci. 2008;15(3):278-85.

29. Li M, Li Z, Yao Y, Jin WN, Wood K, Liu Q, Shi FD, Hao J. Astrocyte-derived interleukin-15 exacerbates ischemic brain injury via propagation of cellular immunity. Proc Natl Acad Sci U S A. 2017;114(3):e396-405.

30. Kraner SD, Norris CM. Astrocyte activation and the Calcineurin/NFAT pathway in cerebrovascular disease. Front Aging Neurosci. 2018;10:287.

31. Liu X, Wu D, Wen S, Zhao S, Xia A, Li F, Ji X. Mild therapeutic hypothermia protects against cerebral ischemia/ reperfusion injury by inhibiting miR-15b expression in rats. Brain Circulation. 2017;3(4):219-26.

32. Fan F, Yang J, Xu Y, Guan S. MiR-539 targets MMP-9 to regulate the permeability of blood-brain barrier in ischemia/ reperfusion injury of brain. Neurochem Res. 2018;43(12):2260-7.

33. Ma J, Bao L, Xia X, Feng Q, Zhou Y, Wang Y, Cao Z. miRNA-128b promotes cerebral infarction by regulating the expressions of BCl-2 and caspase 3. World Neurosurgery. 2018;123:e245-51.

34. Falkowska A, Gutowska I, Goschorska M, Nowacki P, Chlubek D, Baranowska-Bosiacka I. Energy metabolism of the brain, including the cooperation between astrocytes and neurons, especially in the context of glycogen metabolism. Int J Mol Sci. 2015;16(11):25959-81.

35. Geng J, Zhang Y, Li S, Li S, Wang J, Wang H, Aa J, Wang G. Metabolomic Profiling Reveals That Reprogramming of Cerebral Glucose Metabolism Is Involved in Ischemic Preconditioning-Induced Neuroprotection in a Rodent Model of Ischemic Stroke. J Proteome Res. 2018;18(1):57-68.

36. McKenna MC, Scafidi S, Robertson CL. Metabolic alterations in developing brain after injury: knowns and unknowns. Neurochem Res. 2015;40(12):2527-43.

37. Brekke E, Berger HR, Wideroe M, Sonnewald U, Morken TS. Glucose and intermediary metabolism and astrocyte-neuron interactions following neonatal hypoxia-ischemia in rat. Neurochem Res. 2017:42(1):115-32.

38. Xie Z, Cao BQ, Wang T, Lei Q, Kang T, Ge CY, Gao WJ, Hui H. LanCL1 attenuates ischemia-induced oxidative stress by Sirt3-mediated preservation of mitochondrial function. Brain Res Bull. 2018;142:216-23.

39. Yu S, Xu H, Chi X, Wei L, Cheng Q, Yang Y, Zhou C, Ding F. 2-(4-Methoxyphenyl)ethyl-2-Acetamido-2-deoxy-beta-dpyranoside (a Salidroside analog) confers neuroprotection with a wide therapeutic window by regulating local glucose metabolism in a rat model of cerebral ischemic injury. Neuroscience. 2018;391:60-72.

40. Shen X, Li H, Li W, Wu X, Sun Z, Ding X. Telmisartan ameliorates adipoR1 and adipoR2 expression via PPAR-gamma activation in the coronary artery and VSMCs. Biomed Pharmacother. 2017;95:129-36.

41. Zhang Z, Li Y, Yang X, Wang L, Xu L, Zhang Q. Susceptibility of multiple polymorphisms in ADIPOQ, ADIPOR1 and ADIPOR2 genes to myocardial infarction in Han Chinese. Gene. 2018;658:10-7.

42. Lei L, Zhou C, Yang X, Li L. Down-regulation of microRNA-375 regulates adipokines and inhibits inflammatory cytokines by targeting AdipoR2 in non-alcoholic fatty liver disease. Clin Exp Pharmacol Physiol. 2018;45(8):819-31.

43. Zhu P, Huang W. Design, synthesis chalcone derivatives as AdipoR agonist for type 2 diabetes. Chem Biol Drug Design. 2018;92(2):1525-36.

44. Kim Y, Lim JH, Kim MY, Kim EN, Yoon HE, Shin SJ, Choi BS, Kim YS, Chang YS, Park CW. The adiponectin receptor agonist AdipoRon ameliorates diabetic nephropathy in a model of type 2 diabetes. J Am Soc Nephrol. 2018;29(4):1108.

45. Pal China S, Sanyal S, Chattopadhyay N. Adiponectin signaling and its role in bone metabolism. Cytokine. 2018. doi. https://doi.org/10.1016/j.cyto.2018.06.012. [Epub ahead of print].

46. Yuan B, Teng JF. Association between adiponectin receptor 2 gene polymorphisms and cerebral infarction. Genet Mol Res. 2014;13(3):7808-14. 